\title{
Solutions of the Ginsparg-Wilson relation
}

\author{
Ting-Wai Chiu* and Sergei V. Zenkin ${ }^{\dagger}$ \\ Department of Physics, National Taiwan University, Taipei, Taiwan 106, Republic of China
}

(Received 22 June 1998; published 11 February 1999)

\begin{abstract}
We analyze the general solutions of the Ginsparg-Wilson relation for lattice Dirac operators and formulate a necessary condition for such operators to have a nonzero index in the topologically nontrivial background gauge fields. [S0556-2821(99)03405-0]
\end{abstract}

PACS number(s): 11.15.Ha, 11.30.Fs, 11.30.Rd

Recently there have been very interesting developments in the theoretical understanding of chiral symmetry on the lattice. The idea stems from the Ginsparg-Wilson (GW) relation [1] which was derived in 1981 as the remnant of chiral symmetry on the lattice after blocking a chirally symmetric theory with a chirality breaking local renormalization group transformation. The original GW relation is

$$
D \gamma_{5}+\gamma_{5} D=2 a D \gamma_{5} R D
$$

where $D$ is lattice Dirac operator, $R$ is a nonsingular Hermitian operator which is local in position space and trivial in Dirac space, and $a$ is the lattice spacing which reminds us that $D$ becomes chirally symmetric in the continuum limit $a \rightarrow 0$. According to the Nielsen-Ninomiya theorem [2] the chiral symmetry of a local Dirac operator defined on the regular lattice must be broken in order to avoid species doubling. The main advantage of the GW relation is that it introduces chiral symmetry breaking of $D$ in the mildest way [1]. Although it does not ensure the absence of the species doubling, it does incorporate two remarkable properties. try

The first is that the action $A=\bar{\psi} D \psi$ has an exact symme-

$$
\begin{aligned}
& \psi \rightarrow \exp \left[i \theta \gamma_{5}(1-R D)\right] \psi, \\
& \bar{\psi} \rightarrow \bar{\psi} \exp \left[i \theta(\mathbb{1}-D R) \gamma_{5}\right],
\end{aligned}
$$

where $\theta$ is a global parameter, which was discovered by Lüscher [3]. The second is that any operator $D$ satisfying the $\mathrm{GW}$ relation possesses a well defined integer index on a finite lattice $[4,3]$

$$
\lim _{\epsilon \rightarrow 0} \epsilon \sum_{n}\left\langle\bar{\psi}_{n} \gamma_{5} \psi_{n}\right\rangle_{f}=\operatorname{Tr}\left(\gamma_{5} R D\right)=n_{-}-n_{+} \equiv \operatorname{index} D
$$

\footnotetext{
*Email address: twchiu@phys.ntu.edu.tw

${ }^{\dagger}$ Permanent address: Institute for Nuclear Research of the Russian Academy of Sciences, 117312 Moscow, Russia. Email address: zenkin@al20.inr.troitsk.ru
}

where the left-hand side stands for the fermionic average of $\bar{\psi} \gamma_{5} \psi$ calculated with the infinitesimal mass $\epsilon$ added to the operator $D$, and $n_{+}\left(n_{-}\right)$are the number of the zero modes of $D$ with positive (negative) chiralities. This is in contrast to the Wilson-Dirac operator for which the left-hand side (LHS) generally is not an integer on a finite lattice.

It is essentially due to these two properties that such formulations of lattice QCD can possess the attractive features pointed out in Refs. [3-6]. However, only the GW relation itself is not sufficient to guarantee that any $D$ satisfying Eq. (1) must possess exact zero modes with definite chiralities, and reproduce the Atiyah-Singer index theorem on the lattice. In this paper, we analyze general solutions of GW relation and formulate a necessary condition for them to have nonzero indices in topologically nontrivial background gauge fields. We limit our consideration to the operators $D$ satisfying the Hermiticity property

$$
D^{\dagger}=\gamma_{5} D \gamma_{5}
$$

First, we consider the case of nonsingular $D$ which is relevant to topologically trivial gauge field background, except possibly some "exceptional" configurations. Then Eq. (1) is equivalent to the following equation linear in $D^{-1}$ :

$$
\gamma_{5} D^{-1}+D^{-1} \gamma_{5}=2 a \gamma_{5} R
$$

and its general solution can be written in the form

$$
D=\left(1+a D_{c} R\right)^{-1} D_{c}=D_{c}\left(1+a R D_{c}\right)^{-1},
$$

where $D_{c}$ is the chirally symmetric lattice Dirac operator, i.e.,

$$
D_{c} \gamma_{5}+\gamma_{5} D_{c}=0
$$

Thus in the nonsingular case the problem of constructing explicit solutions of $D$ reduces to finding a proper realization of the chirally symmetric operator $D_{c}$. Note that by virtue of the condition (5) and Eq. (8) the operator $D_{c}$ is antiHermitian, and therefore, normal. In order to avoid species doubling for $D$ defined on a regular lattice, $D_{c}$ should be nonlocal. Additional limitations to the form of $D_{c}$ come from the requirement of the locality of $D$. For a more detailed 
discussion on the properties of $D_{c}$ we refer to our paper [7] where a few explicit examples are also presented.

It is interesting to observe that in this case both $D_{c}$ and $D$ can be constructed from a unitary operator $V\left(V^{\dagger}=V^{-1}\right)$ which satisfies the Hermiticity condition

$$
\gamma_{5} V \gamma_{5}=V^{\dagger}
$$

Indeed, for any given chirally invariant $D_{c}$ satisfying Eq. (5), $D_{c}$ is anti-Hermitian, so that $\operatorname{det}\left(a D_{c}+1\right) \neq 0$. Then there exists a unitary operator

$$
V=\left(a D_{c}-1\right)\left(a D_{c}+1\right)^{-1}
$$

satisfying Eq. (9). So $D_{c}$ can be represented as

$$
D_{c}=a^{-1} \frac{1+V}{1-V},
$$

provided that $1-V$ is nonsingular. Substituting Eq. (11) into Eq. (7), we obtain the general solution of Eq. (6) for nonsingular $D$ in terms of the unitary operator $V$ :

$$
\begin{aligned}
D & =a^{-1}[(1+V) R+1-V]^{-1}(1+V) \\
& =a^{-1}(1+V)[R(1+V)+1-V]^{-1} .
\end{aligned}
$$

Note that in contrast to Eq. (11) these expressions make sense even when operator $1-V$ is singular. As we will show later, due to this fact Eqs. (12) and (13) represent a class of solutions of Eq. (1) also for singular $D$, and thus are valid for any gauge configurations.

Let us now consider the case when $D$ is singular, i.e., $\operatorname{det} D=0$, which is the result we would like to have in the topologically nontrivial gauge field background. In this case we are interested in only those $D$ 's which have the possibility to reproduce the index theorem on the lattice, i.e., have a nonzero index in Eq. (4). So we obtain a necessary condition for any solutions of Eq. (1) to possess nonzero indices in topologically nontrivial background gauge fields. We hope that this condition not only serves as a discriminant to rule out any unphysical solutions of GW relation but also can provide guidelines to construct viable solutions of GW relation. We state our result in the following theorem.

Theorem. For any lattice Dirac operator $D$ satisfying hermiticity condition (5) and the GW relation (1), the necessary condition for it to have a nonzero index in the topologically nontrivial gauge background is

$$
\operatorname{det}(1-a D R)=0
$$

or, equivalently,

$$
\operatorname{det}(1-a R D)=0 .
$$

Proof . Assume that $\operatorname{det}(1-a D R) \neq 0$. Then there exists a chirally symmetric normal operator

$$
D_{c}=(1-a D R)^{-1} D .
$$

It is obvious that any zero mode of $D$ is a zero mode of $D_{c}$, and vice versa. Therefore index $D_{c}=\operatorname{index} D$. However, according to the theorem proved in Ref. [8], the index of any chirally symmetric normal Dirac operator is zero, so index $D_{c}=\operatorname{index} D=0$. Since $\operatorname{det}(1-a D R)=\operatorname{det}(1-a R D)$, this completes the proof.

In other words, we have proved that in order the operator $D$ to have nonzero index the chirally invariant operator $D_{c}$ in Eq. (16), and therefore in Eq. (7), should not exist. However, $D$ is still well defined and exists. Equations (11) and (12) suggest a simple interpretation of this seemingly paradoxial situation. As shown in Ref. [9], for any unitary operator $V$ satisfying Eq. (9), if it has real $( \pm 1)$ eigenmodes then these real eigenmodes are chiral and the total chirality of all real eigenmodes must vanish:

$$
n_{+1}^{+}-n_{+1}^{-}+n_{-1}^{+}-n_{-1}^{-}=0,
$$

where $n_{+1}^{+}\left(n_{+1}^{-}\right)$denotes the number of positive (negative) chirality eigenmodes of eigenvalue +1 , while $n_{-1}^{+}\left(n_{-1}^{-}\right)$denotes the number of positive (negative) chirality eigenmodes of eigenvalue -1 . The -1 eigenmodes of $V$ correspond to the zero modes of $D$. Thus, if $D$ has nonzero index $\left(n_{-1}^{-}\right.$ $-n_{-1}^{+} \neq 0$ ), then $n_{+1}^{+}-n_{+1}^{-} \neq 0$ and $V$ has eigenvalue +1 . Then the chirally invariant operator $D_{c}$ in Eq. (11) is no longer defined, while the operator $D$ in Eq. (12) becomes singular but still well defined. Therefore Eq. (12) is indeed a class of general solutions for the GW relation (1) for any gauge configurations.

Finding a unitary operator $V$ which can have eigenvalues +1 and -1 in the topologically nontrivial sectors, however, is a highly nontrivial task. So far we know only one explicit example of $V$ which does satisfy this requirement. It is the unitary operator derived from the overlap formalism [5]:

$$
V=D_{w}\left(D_{w}^{\dagger} D_{w}\right)^{-1 / 2},
$$

where $D_{w}$ is the standard Wilson-Dirac operator but with a negative mass in the range $\left(-2 a^{-1}, 0\right)$. In Ref. [9], it has been demonstrated that this solution of the GW relation indeed reproduces exact zero modes and the index theorem is satisfied exactly on a finite lattice. The zero modes are also in very good agreement with the continuum theory. At this moment we cannot provide another example of $V$ which can satisfy all our requirements.

It is instructive to note that solutions of the GW relation may have a zero index not only because zero modes with opposite chiralities always appear in pairs but also because they may not have any zero modes at all. Consider the naive massless Dirac fermion operator $D_{n}$ on the regular lattice, the random lattice [10], and the random-block lattice [11], respectively. Since $D_{n}$ is chirally symmetric, it can be taken as $D_{c}$ and the Dirac operator $D$ can be constructed from Eq. (7). For any one of these GW-Dirac operators, we do not find any genuine zero modes in any topologically nontrivial sectors. 
To summarize, we have demonstrated that the GW relation does not guarantee the existence of exact zero modes nor the realization of index theorem on the lattice. If a solution of GW relation $D$ in topologically nontrivial sector gives $\operatorname{det}(1-a R D) \neq 0$, and therefore can be expressed in terms of a chirally invariant operator $D_{c}$, its index must be zero, and thus it should be dropped from the list of viable lattice Dirac fermion operators. We note in passing that the necessary condition (14) or (15) is precisely the condition that the gen- erators of the lattice chiral transformations in Eqs. (2) and (3) are singular. The general solution for the GW relation (1) is obtained in Eq. (12).

This work was supported by the National Science Council, R.O.C. under Grant No. NSC87-2112-M002-013. T.W.C. would like to thank Herbert Neuberger for enlightening correspondences. S.V.Z is grateful to members of the Department of Physics at National Taiwan University for the hospitality extended to him during his stay at Taipei.
[1] P. Ginsparg and K. Wilson, Phys. Rev. D 25, 2649 (1982).

[2] H. B. Nielsen and N. Ninomiya, Nucl. Phys. B185, 20 (1981); B193, 173 (1981).

[3] M. Lüscher, Phys. Lett. B 428, 342 (1998).

[4] P. Hasenfratz, V. Laliena, and F. Niedermayer, Phys. Lett. B 427, 125 (1998).

[5] H. Neuberger, Phys. Lett. B 417, 141 (1998); 427, 353 (1998).

[6] P. Hasenfratz, Nucl. Phys. B525, 401 (1998); S. Chan- drasekharan, "Lattice QCD with Ginsparg-Wilson fermions," hep-lat/9805015.

[7] T. W. Chiu, C. W. Wang, and S. V. Zenkin, Phys. Lett. B 438, 321 (1998)

[8] S. V. Zenkin, Phys. Rev. D 58, 057705 (1998).

[9] T. W. Chiu, Phys. Rev. D 58, 074511 (1998).

[10] N. H. Christ, R. Friedberg, and T. D. Lee, Nucl. Phys. B210[FS6], 337 (1982).

[11] T. W. Chiu, Phys. Lett. B 206, 510 (1988). 